\title{
Rhodium nanoparticles and halloysite nanoclay as electrode modifiers for electroanalytical determination of paracetamol
}

\begin{abstract}
A new electrochemical sensor (HNC-Rh-(ImS3-14)/GCE) was built by coating a glassy carbon electrode (GCE) with a suspension made of rhodium nanoparticles stabilized in zwitterionic surfactant (3-(1-tetradecyl-3-imidazolium) propanesulfonate (ImS3-14)) and halloysite nanoclay (HNC). The sensor was characterized by cyclic voltammetry, electrochemical impedance spectroscopy, transmission electron microscopy and profilometry. The modifier was found to enhance the electroactive surface area and lower the charge transfer resistance in comparison to the bare GCE. The proposed sensor was applied in the electrochemical analysis of paracetamol, with a limit of detection of 82.78 $\mathrm{nmol} \mathrm{L}-1$ and a linear range of 0.9 to $10.9 \mu \mathrm{mol} \mathrm{L}-1(\mathrm{r} 2=0.995)$ under optimized conditions (acetate buffer $0.1 \mathrm{~mol} \mathrm{L-1,} \mathrm{pH} 5.0$ employing differential pulse voltammetry). Precision studies revealed the proposed sensor is reproducible on an interday/intraday basis showing low relative standard errors. Paracetamol tablets were selected as target sample and the sensor showed good selectivity and low relative error in comparison to the labelled content ( $\mathrm{RE}=1,67 \%$ ) with recoveries ranging from 107.6 to $123.2 \%$.
\end{abstract}

Keywords: halloysite nanoclay, nanoparticles, paracetamol, rhodium nanoparticles, sensor
Volume 6 Issue 4 - 2017

\author{
Larissa Moreira Ferreira, lolanda Cruz Vieira \\ 'Department of Chemistry, Federal University of Santa \\ Catarina, Brazil \\ 2Department of Teaching Methodology, Federal University of \\ Santa Catarina, Brazil
}

\begin{abstract}
Correspondence: Larissa Moreira Ferreira, Department of Chemistry, Department of Teaching Methodology, Federal University of Santa Catarina, Brazil, Tel 5.54837E+II, Email larisaa.ferreira@ufsc.br
\end{abstract}

Received: November 22, 2017 | Published: December 05, 2017
Antions: $-\mathrm{HNC}$, halloysite nanoclay;

Abbreviations: HNC, halloysite nanoclay; EIS, electrochemical impedance spectrometry; TEM, transmission electron microscopy; GCE, glassy carbon electrode; CV, cyclic voltammetry; LSV, linear sweep voltammetry; SWV, square-wave voltammetry; DPV, differential pulse voltammetry

\section{Introduction}

Despite the known side effects of liver damage, among other issues, paracetamol (N-acetyl-p-aminophenol) continues to be one of the most commonly used drugs worldwide for conditions such as pain and fever and it is often purchased without medical prescription. It is usually administered as a tablet, but it is also available as solutions, suppositories and intravenous preparations. In addition, paracetamol is often taken in association with other drugs like caffeine, tramadol and codeine. ${ }^{1-3}$

Given its chemical, commercial and medical significance, several methods of analysis have been developed to determine its content accurately in multiple kinds of samples. Analytical techniques such as spectrophotometry, ${ }^{4-6}$ chromatography ${ }^{7-8}$ and mass spectrometry ${ }^{9-10}$ have been employed in the determination of paracetamol, as well as electrochemical techniques ${ }^{11-13}$ which offer a reliable, fast and simple method of analysis with no need for complicated sample preparation procedures.

Among other areas of research, electroanalytical sensors have been constructed in order to enhance analytical parameters using diverse components. In this context, nanomaterials are notable for their unique electrical, optical and magnetic properties. ${ }^{14-15}$ Several different nanomaterials have been synthesised and applied in the construction of electrochemical sensors. A series of metallic nanoparticles stabilized in a zwitterionic surfactant (ImS3-14) was found to be suitable for this purpose, due to the metallic nature and small size of the particles and because the stabilizer itself, i.e., the zwitterionic surfactant, can be used as a modifier for electrochemical sensors. ${ }^{16-19}$

Halloysite nanoclay (HNC) also attracts interest not only due to its effect in enhancing analytical parameters for electrochemical sensors, but also because it is widely available, cost effective and biodegradable. $^{20,21}$ The aim of this study was to investigate the suitability of a sensor constructed with a mixture of halloysite nanoclay and rhodium nanoparticles stabilized in zwitterionic surfactant (Rh(ImS3-14)) for paracetamol determination.

\section{Experimental}

\section{Reagents and solutions}

All reagents, that is, paracetamol, starch, cellulose, chloroform, $\mathrm{RhCl}_{2}$, sodium acetate, sodium phosphate, acetic acid, phosphoric acid and sodium hydroxide, were analytical grade (Sigma-Aldrich) and used without further purification. The halloysite nanoclay was purchased from Sigma-Aldrich and contained nanotubes with 30$70 \mathrm{~nm}$ of diameter and $1-3 \mu \mathrm{m}$ of length. All solutions were prepared in ultrapure water obtained from a Milli-Q System (Millipore, USA) with a resistivity of $18.2 \mathrm{M} \Omega \mathrm{cm}^{-1}$. The Rh-(ImS3-14) reverse micelle solution was synthesized according to a procedure previously described in the literature. ${ }^{19}$ The paracetamol tablets were purchased at a local drugstore.

\section{Instrumentation}

The voltammetric experiments were carried out using an Autolab PGSTAT204 potentiostat (Eco Chemie, The Netherlands). The measurements were performed in an electrochemical cell containing $10 \mathrm{~mL}$ of supporting electrolyte using a three-electrode system: platinum wire as the auxiliary electrode, $\mathrm{Ag} / \mathrm{AgCl}\left(3.0 \mathrm{~mol} \mathrm{~L}^{-1}\right)$ as the reference electrode, and the proposed sensor as the working electrode (HNC-Rh-(ImS3-14)/GCE). All measurements were carried out at 
room temperature $\left(25^{\circ} \mathrm{C}\right)$ and no deoxygenation procedures were taken.

Electrochemical impedance spectrometry (EIS) experiments were performed using an Autolab PGSTAT128N potentiostat (Eco Chemie, The Netherlands). For both potentiostats, the software Nova 1.10 was used to collect and evaluate the electrochemical data. Spectrophotometric measurements were performed with a Cary 60 UV-Vis spectrometer (Agilent Technologies, USA). Profilometric measurements were taken on a Dektak XT profilometer (Bruker). Transmission electron microscopy (TEM) analysis was performed on a JEOL JEM-1011 TEM microscope operating at $100 \mathrm{kV}$ at LCME/ UFSC, Florianópolis, Brazil.

\section{Sample preparation}

Ten tablets containing 750mg of paracetamol purchased from a local drugstore were macerated. From the resulting powder an aliquot $(150 \mathrm{mg})$ was dissolved in $2.5 \mathrm{~mL}$ of $0.1 \mathrm{~mol} \mathrm{~L}^{-1} \mathrm{NaOH}, 5 \mathrm{~mL}$ of water were added and the resulting mixture was agitated for $15 \mathrm{~min}$. After the agitation, the mixture was diluted with water in a $10 \mathrm{~mL}$ volumetric flask and the mixture was then filtered. From the liquid part of the filtered mixture, $1 \mathrm{~mL}$ was transferred to a $10 \mathrm{~mL}$ volumetric flask, along with $1 \mathrm{~mL}$ of $0.1 \mathrm{~mol} \mathrm{~L}^{-1} \mathrm{NaOH}$, and the volume completed with water. The resulting solution was transferred to an amber flask and kept under refrigeration.

\section{HNC-Rh-(ImS3-I4) sensor construction}

An aliquot $(100 \mu \mathrm{L})$ of the $\mathrm{Rh}$-( $\operatorname{ImS3}-14)$ solution was mixed with $100 \mathrm{mg}$ of halloysite nanoclay and the resulting suspension (HNCRh-(ImS3-14)) was stirred using a vortex mixer for $1 \mathrm{~min}$. A glassy carbon electrode (GCE) was polished using an aqueous alumin a $(0.05 \mu \mathrm{m})$ suspension for $2 \mathrm{~min}$. It was then immersed in deionized water and sonicated for $5 \mathrm{~min}$. The electrode was dried and $2 \mu \mathrm{L}$ of the (HNC-Rh-(ImS3-14)) suspension were deposited on the clean surface employing the drop coating method. The solvent (chloroform) was left to evaporate at room temperature and the modified GCE was used as the working electrode.

\section{Preparation and characterization of the (HNC-Rh- (ImS3-I4)) dispersion}

The (HNC-Rh-(ImS3-14)) suspension was characterized by TEM. The sample for TEM analysis was prepared by deposition of the chloroform (HNC-Rh-(ImS3-14)) dispersion on a carbon-coated copper grid. Figure 1A shows the halloysite nanoclay nanotubes well dispersed in the sample as well as the size range as predicted by the manufacturer. At a greater magnification (Figure 1B), it can be observed that the surfactant-stabilized Rh NPs are also well dispersed in the halloysite nanoclay dispersion.



Figure I TEM images of (HNC-Rh-(ImS3-I4)) suspension at lower magnification, in which halloysite nanoclay nanotubes can be observed $(A)$ and at higher magnification, in which an halloysite nanoclay nanotube can be seen surrounded by Rh NPs stabilized in ImS3-I4 (B).
The histogram constructed from the measurement of the $\mathrm{Rh}$ nanoparticles observed in the TEM images (Figure 2) shows that they are very small, despite being polydispersed in terms of size. They are roughly spherical in shape with diameters ranging from 5 to $11 \mathrm{~nm}$, based on the measurement of around 300 nanoparticles. The thickness of the (HNC-Rh-(ImS3-14)) film on the GCE was determined, in triplicate, by profilometry measurements. Under the optimum conditions of construction, the average thickness obtained for the film was $7.80 \pm 2.43 \mu \mathrm{m}$.

\section{Results and discussion}

\section{Electrochemical response}

An electrochemical impedance spectroscopy (EIS) assay was performed in order to compare the resistance of the surface of the proposed sensor to that of Rh-(ImS3-14)/GCE and bare GCE in the presence of $\mathrm{Fe}(\mathrm{CN})_{6}^{3-4-4}$ (Figure 3). From the semicircular portion of the Nyquist plots, which corresponds to the charge transfer resistance $\left(\mathrm{R}_{\mathrm{ct}}\right)$, the effect of each modifier on this process can be observed. The $\mathrm{R}_{\mathrm{ct}}$ values calculated for the electron transfer resistance were $1.74 \mathrm{k} \Omega$ for the bare GCE, $614 \Omega$ for the Rh-(ImS3-14)/GCE and $409 \Omega$ for the HNC-Rh-(ImS3-14)/GCE.



Figure 2 Size distribution of the Rh nanoparticles.



Figure 3 Nyquist plots of EIS results for bare GCE, Rh-(ImS3-14)/GCE and HNC-Rh-(ImS3-I4)/GCE obtained with $\mathrm{Fe}(\mathrm{CN})_{6}^{3-44} 5 \mathrm{mM}$, open circuit mode, $10 \mathrm{mV}$ amplitude and frequency range of $0.1-100000 \mathrm{~Hz}$.

Thus, $\mathrm{R}_{\mathrm{ct}}$ is lower when using the Rh-(ImS3-14)/GCE system in comparison to the bare GCE and the addition of halloysite nanoclay causes a further decrease in $\mathrm{R}_{\mathrm{ct}}$, which is consistent with results reported in the literature. ${ }^{21}$ The electrode area was determined by cyclic voltammetry at scan rates varying from 10 to $200 \mathrm{mV} \mathrm{s}^{-1}$ using


Randles-Sevcik formula:

$$
\mathrm{I}_{\mathrm{p}}=2.69 \times 10^{5} A D^{1 / 2} n^{3 / 2} C v^{1 / 2}
$$


Where $I_{p}$ refers to the peak current, $n$ is the number of electrons transferred, $\mathrm{A}$ is the surface area of the electrode, $\mathrm{D}$ is the diffusion coefficient $\left(\mathrm{D}=6.7 \times 10^{-6} \mathrm{~cm}^{2} \mathrm{~s}^{-1}\right),{ }^{22} \mathrm{C}$ is the concentration $\left(9.9 \times 10^{-}\right.$ $\left.{ }^{5} \mathrm{~mol} \mathrm{~L}^{-1}\right), \mathrm{n}$ is the number of electrons $(\mathrm{n}=1)$. From the slope of the plot $\mathrm{I}_{\mathrm{p}} \mathrm{vS} \mathrm{\textrm {v } ^ { 1 / 2 }}$, the electroactive area was calculated for the proposed sensor $\left(\mathrm{A}=0.0406 \mathrm{~cm}^{2}\right)$ to and for bare $\mathrm{GCE}\left(\mathrm{A}=0.0217 \mathrm{~cm}^{2}\right)$. Hence, it can be concluded that the proposed sensor almost doubles the electroactive area of the electrode.

The effect on the paracetamol peak current of each component on the GCE surface was also evaluated. It can be observed from Figure 4 that the addition of each component contributes to the enhancement of the peak current. The peak displacement observed when the surfactant (ImS3-14) and the Rh NPs stabilized in the surfactant are added, is slightly reversed by the presence of the halloysite nanoclay, which indicates a catalytic effect on the paracetamol oxidation. Given the results obtained from the EIS study and the electrode area determination, it can be concluded that the enhancement of the oxidation peak is due to a combination of a lower charge transfer resistance and the increased electroactive surface area due to the presence of the modifiers.



Figure 4 Peak currents for oxidation of paracetamol for different modifiers on the GCE surface for $5.96 \mu \mathrm{mol} \mathrm{L}^{-1}$ of paracetamol in $\mathrm{pH} 5.0$ (acetate buffer $0.1 \mathrm{~mol} \mathrm{~L}^{-1}$ ) using DPV (scan increment of $6 \mathrm{mV}$, pulse amplitude of $130 \mathrm{mV}$ and time of $0.8 \mathrm{~s})$.

\section{Optimization of sensor construction and experimental parameters}

Studies were performed in order to determine the optimum amount of halloyiste nanoclay in relation to the (Rh-(ImS3-14)) solution as well as the number of layers of the suspension in the electrode surface that provides the highest electrochemical response for the paracetamol oxidation. Three different dispersions were prepared by adding varied volumes of the (Rh-(ImS3-14)) solution to a flask containing a fixed amount $(1 \mathrm{mg})$ of halloysite nanoclay. The results show that the best ratio was obtained with the dispersion comprised of $100 \mu \mathrm{L}$ of (Rh(ImS3-14)) solution per $1 \mathrm{mg}$ of halloysite nanoclay. Thus, this ratio was applied in the subsequent experiments.

The number of layers deposited on the GCE surface in relation to oxidation peak current was also investigated. It was found that with more than one layer of solution the electrochemical response decreased, probably due to a blocking effect. Consequently, experiments were performed applying one layer comprised of $2 \mu \mathrm{L}$ of the (HNC-Rh-(ImS3-14)) suspension onto the surface of the GCE.

Cyclic voltammetry $(\mathrm{CV})$ was employed to investigate the influence of the $\mathrm{pH}$ on the peak currents for paracetamol oxidation (Figure 5A). The $\mathrm{pH}$ was varied from 2.0 to $7.0 \mathrm{using} 0.1 \mathrm{~mol} \mathrm{~L}^{-1}$ acidified acetate buffer solution $(\mathrm{pH} 2.0-3.0), 0.1 \mathrm{~mol} \mathrm{~L}^{-1}$ acetate buffer solution $(\mathrm{pH}$
4.0-5.0) and $0.1 \mathrm{~mol} \mathrm{~L}^{-1}$ phosphate buffer solution ( $\left.\mathrm{pH} 6.0-7.0\right)$. The optimum response was obtained in acetate buffer $\mathrm{pH} 5.0$, where the potential was found to be $0.56 \mathrm{~V}$ and, therefore, this was chosen as the supporting electrolyte for subsequent studies.

The relation between the peak potential and $\mathrm{pH}$ (Figure 5B) was found to be linear $\left(\mathrm{r}^{2}=0.98\right)$ with a slope of $-37.03 \mathrm{mV} / \mathrm{pH}$, indicating a complex electron transfer reaction with an uneven number of protons and electrons exchanged, as reported previously in the literature. ${ }^{23}$ From the cyclic voltammograms (Figure 5C) it can also be concluded that the paracetamol in this system shows an evident oxidation peak and the reduction peak is almost absent, behaviour consistent with an irreversible system. For verification, the potential was plotted against the logarithm of the scan rate, which gave a straight line $\left(\mathrm{E}_{\mathrm{p}}=-\right.$ $0.52+0.04 \log \mathrm{v})$. Therefore, considering the observed variation in the potential in relation to the peak potential, the irreversibility of the system was confirmed.


Figure 5 Peak currents for oxidation of paracetamol in relation to $\mathrm{pH}(\mathrm{A})$, potential variation in relation to $\mathrm{pH}(\mathrm{B})$ and the respective voltammograms (C).

The plot of the logarithm of the anodic peak current vs. logarithm of the scan rate provided a straight line $\left(\log i_{p}=-6.73+0.47 \log v\right)$ with a slope of 0.47 . It can thus be concluded that the system is diffusion-controlled, since the value obtained for the slope is close to the theoretical value of 0.5 for a purely diffusion-controlled processes in which the analyte diffuses to the electrode surface due to a concentration gradient. ${ }^{24}$

In order to determine the best set of parameters to perform the analytical determination, three electroanalytical techniques - linear sweep voltammetry (LSV), square-wave voltammetry (SWV) and differential pulse voltammetry (DPV) were investigated and optimized. For the LSV evaluation, the parameter investigated was the scan rate $\left(10,25,50\right.$ and $\left.75 \mathrm{mV} \mathrm{s}^{-1}\right)$. The scan rate of $25 \mathrm{mV} \mathrm{s}^{-1}$ was selected since this provided the greatest response and the best peak profile.

For the SWV optimization, three parameters were studied: scan increment $(1-7 \mathrm{mV})$, pulse amplitude $(10-90 \mathrm{mV})$ and frequency $(10-$ $40 \mathrm{~Hz}$ ). Noise was observed at higher values, thus limiting the study range. A scan increment of $5 \mathrm{mV}$, pulse amplitude of $60 \mathrm{mV}$ and frequency of $10 \mathrm{~Hz}$ were selected as the optimized parameters, since these values provided the best results in terms of peak current and voltammetric profile.

Lastly, the DPV technique and its parameters were assessed: scan increment $(1-10 \mathrm{mV})$, pulse amplitude $(10-170 \mathrm{mV})$ and time $(0.1$ $0.7 \mathrm{~s})$. The best results were obtained at a scan increment of $6 \mathrm{mV}$, pulse amplitude of $130 \mathrm{mV}$ and time of $0.8 \mathrm{~s}$. The figures of merit for each technique are shown in Table 1. The limits of detection and quantification were calculated according to the following equations:

$$
\mathrm{LOD}=3 \mathrm{~s} / \mathrm{m} ; \mathrm{LOQ}=10 \mathrm{~s} / \mathrm{m}
$$

Where $\mathrm{s}$ is the standard deviation of the y-intercept and $\mathrm{m}$ is the slope of the calibration curve. From the data observed in Table 1, it 
can be concluded that DPV provides the lowest limit of detection (LOD) and limit of quantification (LOQ) for the proposed sensor in the paracetamol determination, a result consistent with the literature. ${ }^{25}$ The calibration curve obtained using this technique is shown in Figure 6.

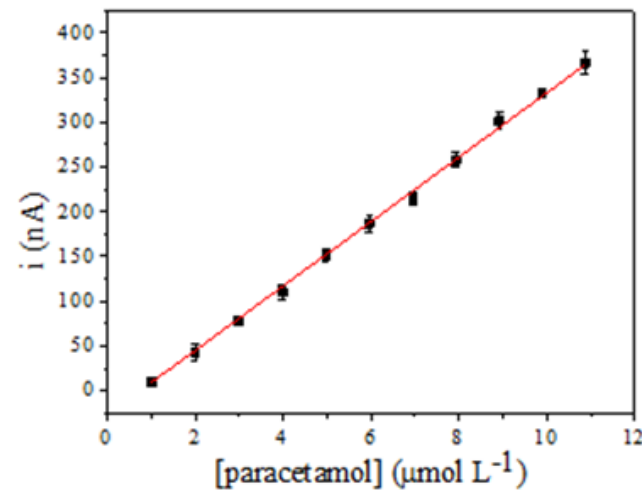

Figure 6 Calibration curve for paracetamol using the proposed sensor with DPV paracetamol in $\mathrm{pH} 5.0$ (acetate buffer $0.1 \mathrm{~mol} \mathrm{~L}^{-1}$ ) using DPV (scan increment of $6 \mathrm{mV}$, pulse amplitude of $130 \mathrm{mV}$ and time of $0.8 \mathrm{~s}$ ).

\section{Precision studies}

Repeatability was evaluated with interday and intraday essays in acetate buffer $\left(0.1 \mathrm{~mol} \mathrm{~L}^{-1}, \mathrm{pH} 4.0\right)$ containing $5.96 \mu \mathrm{mol} \mathrm{L}^{-1}$ of paracetamol using DPV (increment $6 \mathrm{mV}$, pulse amplitude $130 \mathrm{mV}$ and time $0.8 \mathrm{~s})$. Interday repeatability was assessed by evaluating the response of 10 different sensors built with three different GCEs in assays carried out on the same day. For each measurement a new sensor was constructed and the RSD was $5.57 \%$. Intraday studies were carried out under the same conditions as the interday studies, performing 10 experiments on 3 different days with three different GCEs, making a total of 18 measurements. The RSD was $9.97 \%$. From the results, it can be concluded that the proposed sensor has good repeatability.

\section{Interference studies}

Experiments were performed aiming to determine the interference of other compounds in the analysis of paracetamol using the proposed sensor, by comparing the response for paracetamol when adding other substances in fixed amounts. The compounds selected were cellulose and starch, which are commonly employed as excipients. They were tested in the proportions of 1:1, 1:5 and 1:10, in relation to the amount of paracetamol, and they did not interfere with the peak currents for paracetamol due to their non-electroactive nature.

Caffeine, a compound commonly present in pharmaceutical preparations with paracetamol, was also tested for selectivity under the same conditions described for the previously mentioned substances. The results show that the interference of caffeine in relation to the peak currents for paracetamol is $<10 \%$.

Based on these results, it can be concluded that the proposed method provides selectivity for the sample studied as well as for samples containing caffeine in their composition.

Table I Figures of merit for different electroanalytical techniques using the proposed sensor

\begin{tabular}{lllll}
\hline Technique & Analytical Equation & R2 & LOD $(\boldsymbol{\mu m o l}$ L-I $)$ & LOQ $(\boldsymbol{\mu m o l ~ L - I )}$ \\
\hline LSV & $\mathrm{i}=1.15 \times 10-2[$ paracetamol] $-5.51 \times 10-9 \pm 1.33 \times 10-9$ & 0.998 & 3.48 & 11.59 \\
SWV & $\Delta \mathrm{i}=2.48 \times 10-2[$ paracetamol] $-2.92 \times 10-8 \pm 3.11 \times 10-9$ & 0.999 & 0.22 & 0.75 \\
DPV & $\mathrm{i}=3.65 \times 10-2[$ paracetamol] $-3.11 \times 10-8 \pm 9.92 \times 10-8$ & 0.999 & 0.082 & 0.28 \\
\hline
\end{tabular}

$\mathrm{LOD}=3 \mathrm{~s} / \mathrm{m} \mathrm{LOQ}=10 \mathrm{~s} / \mathrm{m}$

Table 2 Comparison of the analytical performance of the different modified electrodes

\begin{tabular}{llll}
\hline Modified Electrode & Detection Limits $(\boldsymbol{\mu m o l ~ L - ~} \mathbf{I})$ & Sensitivity $(\boldsymbol{\mu} \mathbf{A} / \boldsymbol{\mu m o l ~ L - ~ I )}$ & Refs. \\
\hline Fe2O3/SNO2 [a] & 0.2 & 7.43 & 26 \\
Co(II)-zeolite A [b] & 0.04 & 0.017 & 27 \\
Dendrimer/ & 0.24 & 0.013 & 28 \\
PtNPs/Pt [c] & & & \\
DTDF/CNTs [d] & 0.46 & 0.11 & 13 \\
Cd(OH)2-rGO [e] & 0.08 & 24.452 & 29 \\
fCNT/PMG/CE [f] & 4.3 & 3640 & 30 \\
(MWCNTs-G4.0)6 [g] & 0.1 & 0.28 & 31 \\
GQDs [h] & 0.15 & 0.0782 & 32 \\
HNC-Rh-(ImS3-I4) & 0.082 & 0.036 & This study \\
\hline
\end{tabular}

\section{Analytical application of the proposed sensor}

In order to evaluate the accuracy of the proposed method, an analytical determination was performed employing paracetamol tablets purchased in a local drugstore. The result obtained for the paracetamol content was $747.1 \pm 3.57 \mathrm{mg}$. Thus, in comparison to the expected value of $750 \mathrm{mg}$, the relative error was $1.67 \%$, verifying that the proposed method has good accuracy. The percentage recovery ranged from 107.6 to $123.2 \%$, which demonstrates the satisfactory accuracy of the proposed method.

\section{Conclusion}

The proposed sensor was found to be suitable for paracetamol determination since it provides peak enhancement due to a synergic effect between the Rh-(ImS3-14) nanoparticles and the HNC. It also exhibits good analytical parameters such as precision and accuracy. In addition, it can be concluded that the HNC-Rh-(ImS3-14) sensor presents good sensitivity and a low limit of detection in comparison to results recently published for similar studies (Table 2). Given the fact that the sensor preparation is fast, easy and yields good analytical parameters, this represents a suitable method for paracetamol determination. ${ }^{25-32}$

\section{Acknowledgements}

This study was funded by FAPESC/CNPq (Process 2807/ 2012 PRONEM). 


\section{Conflicts of interest}

The authors declare that they have no conflict of interest.

\section{Funding}

None.

\section{References}

1. Association AP. Diagnostic and statistical manual of mental disorders (DSM-5®), American Psychiatric Pub, USA. 2013.

2. Kaufman DW, Kelly JP, Battista DR, et al. Relation of Health Literacy to Exceeding the Labeled Maximum Daily Dose of Acetaminophen. Am J Prev Med. 2016;50(6):e183-e190.

3. Blieden M, Paramore LC, Shah D, et al. A perspective on the epidemiology of acetaminophen exposure and toxicity in the United States. Expert Rev Clin Pharmacol . 2014;7(3):341-348.

4. Jibril F, Sharaby S, Mohamed A, et al. Intravenous versus Oral Acetaminophen for Pain: Systematic Review of Current Evidence to Support Clinical Decision-Making. Can J Hosp Pharm. 2015;68(3):238-247.

5. Hanaee J. Simultaneous determination of acetaminophen and codeine in pharmaceutical preparations by derivative spectrophotometry. Pharmaceutica Acta Helvetiae . 1997;72(4):239-241.

6. Inés Toral M, Jorge Rivas, Marta Saldías, et al. Simultaneous determination of acetaminophen and tramadol by second derivative spectrophotometry. Journal of the Chilean Chemical Society. 2008;53(2):1543-1547.

7. Souri E, Nasab SAM, Amanlou M, et al. Development and validation of a rapid derivative spectrophotometric method for simultaneous determination of acetaminophen, ibuprofen and caffeine. Journal of Analytical Chemistry. 2015; 70(3): 333-338.

8. Bose D, Durgbanshi A, Martinavarro-Domínguez A, et al. Rapid Determination of Acetaminophen in Physiological Fluids by Liquid Chromatography Using SDS Mobile Phase and ED Detection. Journal of Chromatographic Science. 2005;43(6):313-318.

9. Cunha RR, Chaves SC, Ribeiro MMAC, et al. Simultaneous determination of caffeine, paracetamol, and ibuprofen in pharmaceutical formulations by high-performance liquid chromatography with UV detection and by capillary electrophoresis with conductivity detection. Journal of Separation Science. 2015;38(10):1657-1662.

10. Williams JP, Scrivens JH. Rapid accurate mass desorption electrospray ionisation tandem mass spectrometry of pharmaceutical samples. Rapid Communications in Mass Spectrometry. 2005;19(24):3643-3650.

11. Auppila TJ, Wiseman JM, Ketola RA, et al. Desorption electrospray ionization mass spectrometry for the analysis of pharmaceuticals and metabolites. Rapid Communications in Mass Spectrometry. 2006;20(3):387-392.

12. Miner DJ, Rice JR, Riggin RM, et al. Voltammetry of acetaminophen and its metabolites. Analytical Chemistry . 1981;53(14):2258-2263.

13. Zen JM, Ting YS. Simultaneous determination of caffeine and acetaminophen in drug formulations by square-wave voltammetry using a chemically modified electrode. Analytica Chimica Acta 1997;342(2-3):175-180.

14. Mazloum-Ardakani M, Zokaie M, Khoshroo A. Carbon nanotube electrochemical sensor based on and benzofuran derivative as a mediator for the determination of levodopa, acetaminophen, and tryptophan. Ionics. 2015;21(6):1741-1749.

15. Campbell F, Compton R. The use of nanoparticles in electroanalysis: an updated review. Anal Bioanal Chem . 2010;396(1):241-259.
16. Luo X, Morrin A, Killard AJ, et al. Application of Nanoparticles in Electrochemical Sensors and Biosensors. Electroanalysis. 2006;18(4):319-326.

17. Cadorin Fernandes S, Dutra de Souza F, Silveira de Souza B, et al. Gold nanoparticles dispersed in zwitterionic surfactant for peroxidase immobilization in biosensor construction. Sensors and Actuators B: Chemical . 2012;173:483-490.

18. Zapp E, Souza FD, Souza BS, et al. Analyst . 2013;138; 509-517.

19. Souza BS, Leopoldino EC, Tondo DW, et al. Imidazolium-based zwitterionic surfactant: a new amphiphilic Pd nanoparticle stabilizing agent. Langmuir. 2011;28(1):833-840.

20. Fiedler HD, Drinkel EE, Orzechovicz B, et al. Simultaneous nondestructive analysis of palladium, rhodium, platinum, and gold nanoparticles using energy dispersive X-ray fluorescence. Anal Chem. 2013;85(21):10142-10148.

21. Sadegh-Hassani F, Mohammadi Nafchi A. Preparation and characterization of bionanocomposite films based on potato starch/ halloysite nanoclay. International Journal of Biological Macromolecules. 2014;67: 458-462.

22. Sanghavi BJ, Hirsch G, Karna SP, et al. Potentiometric stripping analysis of methyl and ethyl parathion employing carbon nanoparticles and halloysite nanoclay modified carbon paste electrode. Analytica Chimica Acta. 2012;735:37-45.

23. Liang M, Jin F, Liu R, et al. Enhanced electrochemical detection performance of multiwall carbon nanotubes functionalized by aspartame. Journal of Materials Science. 2013;48(16):5624-5632.

24. Goyal RN, Gupta VK, Oyama M, et al. Differential pulse voltammetric determination of paracetamol at nanogold modified indium tin oxide electrode. Electrochemistry Communications. 2005;7(8):803-807.

25. Bard AJ, Faulkner LR. Electrochemical Methods: fundamentals and applications. John Wiley \& Sons, New York, USA. 2001.

26. Molina A, Laborda E, Martínez-Ortiz F, et al. The use of differential pulse voltammetries to discriminate between the Butler-Volmer and the simple Marcus-Hush models for heterogeneous electron transfer: The electro-reduction of europium (III) in aqueous solution. Journal of Electroanalytical Chemistry. 2011;659(1):12-24.

27. Taei M, Shavakhi M, Hadadzadeh H, et al. Simultaneous determination of epinephrine, acetaminophen, and tryptophan using Fe2O3(0.5)/ $\mathrm{SnO} 2(0.5)$ nanocomposite sensor. Journal of Applied Electrochemistry. 2015;45(2):185-195.

28. Ahmadpour-Mobarakeh L, Nezamzadeh-Ejhieh A. A zeolite modified carbon paste electrode as useful sensor for voltammetric determination of acetaminophen. Materials Science and Engineering: C. 2015;49(1):493-499.

29. Armada MPG, Vallejo E, Villena C, et al. New acetaminophen amperometric sensor based on ferrocenyl dendrimers deposited onto Pt nanoparticles. Journal of Solid State Electrochemistry. 2016;20(6):1551-1563.

30. Sakthivel M, Sivakumar M, Chen SM, et al. Reduced Graphene Oxide Non-covalent Functionalized with Zinc Tetra Phenyl Porphyrin Nanocomposite for Electrochemical Detection of Dopamine in Human Serum and Rat Brain Samples. Electroanalysis. 2016;28(9):2126-2135.

31. Barsan MM, Toledo CT, Brett CMA. New electrode architectures based on poly(methylene green) and functionalized carbon nanotubes: Characterization and application to detection of acetaminophen and pyridoxine. Journal of Electroanalytical Chemistry. 2015;736(1):8-15.

32. Zhang Y, Liu X, Li L, et al. An electrochemical paracetamol sensor based on layer-by-layer covalent attachment of MWCNTs and a G4.0 PAMAM modified GCE. Analytical Methods. 2016;8:2218-2225.

33. Zhao C, Liu ZE, Xu W, et al. A glassy carbon electrode based on graphene quantum dots (GQDs) for simultaneous detection of acetaminophen and ascorbic acid. Analytical Methods . 2015;7(20):8877-8881. 